\title{
FUZZY SUPPLY CHAIN COORDINATION MECHANISM WITH IMPERFECT QUALITY ITEMS
}

\author{
Shukuan $\mathrm{LIU}^{1}$, Jie $\mathrm{GAO}^{2}$, Zeshui $\mathrm{XU}^{3 *}$ \\ ${ }^{1}$ Antai School of Economics and Management, Shanghai Jiaotong University, Shanghai 200052, China \\ ${ }^{2}$ Institute for Disaster Management and Reconstruction, Sichuan University, Chengdu 610207, China \\ ${ }^{3}$ Business School, Sichuan University, Chengdu, Sichuan 610064, China
}

Received 1 February 2018; accepted 31 July 2018

\begin{abstract}
We study the supply chain (SC) returning strategy and quantity discount coordination under the condition of product quality defects. We assume that the demand is a triangular fuzzy number (TFN), considering the SC coordination problem consisting of a manufacturer and a retailer. The decentralized SC coordination model and the integrated SC coordination model under a fuzzy environment are established respectively. The fuzzy set theory is used to study the manufacturer's quantity discount and the retailer's coordination of return policy. The signed distance is used as the ranking method to find the optimal order quantity in SC, and the optimization theory is used to maximize the participants' profits. We first demonstrate that the retailer's profit will be reduced in a typical integrated channel, and then we propose a quantitative discount return policy to coordinate the profits of the manufacturer and the retailer. Finally, the coordination steps are designed, and the manufacturer's return policy is given. Meanwhile, some illustrative cases are provided to illustrate the feasibility of the proposed model.
\end{abstract}

Keywords: supply chain, uncertain demand, imperfect quality, return policy, quantity discounts, coordination mechanism, signed distance, fuzzy set theory.

JEL Classification: D81, G41.

\section{Introduction}

Some scholars and practitioners have focused on coordinating prices and order quantities between manufacturers and the retailers in supply chain management (SCM). "Each company in a supply chain (SC) must perform a precise set of actions to achieve optimal SC performance. However, the main goal of each company is its own profit. Therefore, a superior SC needs to coordinate different incentive mechanisms" (Cachon, 2003). Return policy (Emmons \& Gibert, 1998), revenue sharing (Cachon \& Lariviere, 2000), and quantity discounts (Dolan, 1987) are common tools for coordinating conflicts between manufacturers and the retailers. In

*Corresponding author. E-mail: xuzeshui@263.com

This is an Open Access article distributed under the terms of the Creative Commons Attribution License (http://creativecommons. org/licenses/by/4.0/), which permits unrestricted use, distribution, and reproduction in any medium, provided the original author and source are credited. 
most existing literature, goods or products are generally considered to be perfect for ease of calculation and analysis. In fact, due to the limitations of production technology or human error in the production process of workers, some products may not be of good quality. For example, improperly cut garments, incomplete prints, unstable electronic products, etc.

Salameh and Jaber (2000) firstly extended the traditional economic order quantity (EOQ) and the economic production quantity (EPQ) models by considering imperfect quality items. Based on this, Maddah and Jaber (2008) proposed a new model to correct possible deficiencies in their previous work. Jaber, Goyal, and IMran (2008) also extended the model by assuming that the percentage of defects per lot was reduced based on the learning curve. Later on, Chung, Her, and Lin (2009) established a new inventory model with two warehouses and considered imperfect quality simultaneously. Khan, Jaber, and Wahab (2010), Khan, Jaber, and Bonney (2011) extended Salameh and Jaber's (2000) EOQ model where is learning in inspection. Recently, Hsu, J. T. and Hsu, L. F. $(2013,2014)$ have developed a new EOQ model with imperfect quality items, sales returns, incorrect inspections and shortages. Wang, Wee, Cheng, Wen, and Cárdenas-Barrón (2015) proposed an EOQ model for partial out-of-stock and screening constraints for imperfect quality items.

All the above studies assume that the customer demand is uncertain, which involves a probability distribution. This assumption seems to be restrictive. In the real world, statistics are lacking for new products. Some researchers have applied fuzzy set theory (FST) (Zadeh, 1965) to manage imperfect quality items. For example, Chang (2004) extended the EOQ model of Salameh and Jaber's (2000) with imperfect quality, fuzzy defective quality, and fuzzy annual demand. Chen, Wang, and Chang (2007) used the function principle to manipulate arithmetical operations and employed the Kuhn-Tucker conditions to obtain the optimal EPQ of a fuzzy production inventory model. Roy and Samanta (2009), Roy, Maity, kar, and Maiti (2009) developed a fuzzy continuous review inventory model for degraded items and discussed the situation where there is no shortage, the demand rate is unchanging and the cycle time is undetermined. Hu, Zheng, Guo, and Ji (2010) constructed a fuzzy stochastic inventory model, whose production process was not perfect. Ameli, Mirzazadeh, and Shirazi (2011) developed a new inventory model to determine ordering strategies for imperfect items with ambiguous imperfections under fuzzy discounts and inflation conditions. Recently, Mahata and Goswami (2013) used two types of trapezoidal and triangular fuzzy numbers (FNs) to consider an inventory models of items with imperfect quality and shortage in the fuzzy environments. Bhaya, Pal, and Nayak (2014) discussed intuitionistic fuzzy optimization techniques in the EOQ model, in which demand, holding costs, and ordering costs are all FNs, and the quality of items were incomplete. Kazemi, Olugu, Abdul-Rashid, and Ghazilla (2015a), Kazemi, Shekarian, Cárdenas-Barrón, and Olugu (2015b) proposed a new fuzzy EOQ model for quality imperfections with out-of-stock and fuzzy learning. Das, De, and Sana (2015), and De and Sana (2015) also did the research on the EOQ models in fuzzy environment.

With the fierce market competition, the manufacturers and the retailers must work together to meet the harsh customer needs, and more investigations on SC coordination with imperfect quality items have been conducted. Chen and Kang (2010) studied an integrated supplier-buyer model that considers trade credit and imperfect quality. Lin (2010), Lin and Yeh (2010) has developed a new inventory model for products with defective quality and 
quantity discounts, and the buyer can influence its suppliers. Sana (2011) proposed an integrated production inventory model for defective quality items in a three-tier SC. Rad, Khoshalhan, and Glock (2014) developed a supplier-purchaser integrated SC model whose production was imperfect. Taleizadeh, Noori-daryan, and Tavakkoli-Moghaddam (2015) proposed an economic production and inventory model in the SC, in which the products quality was incomplete and the inspections were performed when the defective products were repurchases. Pal, Mahapatra, and Samanta (2016) developed a joint EPQ model for products with random demand and defective quality.

There are few papers on SC coordination of imperfect quality products using FST. Yu and Jin (2011) studied the return strategy model with demand ambiguity and information asymmetry. Zheng, Liu, and Wang (2010) proposed two fuzzy models for poor quality newsboy problem in decentralized and centralized systems. Hu et al. (2010) established a fuzzy stochastic inventory model, whose production processes were incomplete and time was random. Manna, Dey, and Mondal (2014) in the fuzzy rough environment considered the product reliability and the transformation of defective products in the three-layer SC, then an imperfect production inventory model was developed. Kurdhi, Lestari, and Susanti (2015) proposed a fuzzy collaborative SC inventory model in which controllable setup cost and service level for defective products were constrained. Kumar, Tiwari, and Goswami (2016) developed a two-level comprehensive procurement production model for the SC under fuzzy stochastic framework.

We use FST to study the return strategy with quantity discount in SC coordination. We first show that the retailer's profit will decrease within the typical integrated channel. Then, based on the optimal order quantity (OQ) within the integrated channel, we propose a return policy with quantity discount to coordinate the profits of the manufacturer and the retailer. Finally, we employ the optimization theory to maximize the profits of participants and give cases to exemplify the feasibility of the model.

The rest of the paper is organized as follows: Section 1 introduces some fuzzy operations and fuzzy representations. Section 2 introduces some assumptions and notations. Section 3 proposes a coordination mechanism with return policy and quantity discount. In Section 4, some numerical cases are provided to exemplify our model. The paper ends with some conclusions in last section.

\section{Preliminaries}

Zadeh's (1965) FST has a wide range of application in the management field, such as inventory management, supply planning and supplier elections, and has proven to be a suitable means of model uncertainty. In what follows, we give a simple introduction to some wellknown fuzzy operations and the decomposition principle.

Definition 1 (Zimmermann, 2011). For $0 \leq \alpha \leq 1$, the FN $\tilde{a}_{\alpha}$ is defined on $R=(-\infty, \infty)$. If the membership function (MF) of $\tilde{a}_{\alpha}$ is given by

$$
\operatorname{mf} \mu_{\tilde{a}_{\alpha}}(x)= \begin{cases}\alpha, & x=a, \\ 0, & x \neq a,\end{cases}
$$

then $\tilde{a}_{\alpha}$ is called an $\alpha$-level fuzzy point. 
Remark 1. when $\alpha=1$, the MF of $\tilde{a}_{1}$ becomes a characteristic function (CF), i.e., if $x=a$, then $\mu_{\tilde{a}_{1}}=1$, and if $\mu_{\tilde{a}_{1}}=0$, then $x \neq a$. Under this circumstance, the fuzzy point $\tilde{a}_{1}$ is the same as the real number $a \in R$.

Definition 2 (Zimmermann, 2011). Let the FN $\tilde{A}=[a, b, c]$, where $a<b<c$ and $a, b, c \in R=$ $(-\infty, \infty)$. If the MF of $\tilde{A}$ is given by

$$
\mu_{\tilde{A}}(x)= \begin{cases}l(x)=\frac{x-a}{b-a}, & a \leq x \leq b, \\ r(x)=\frac{c-x}{c-b}, & b \leq x \leq c, \\ 0, & \text { otherwise }\end{cases}
$$

then $\tilde{A}$ is called a triangular fuzzy number (TFN).

Definition 3 (Yao $\& \mathrm{Wu}, 2000$ ). For $0 \leq \alpha \leq 1$, if the MF of the FN $\left[a_{\alpha}, b_{\alpha}\right]$ on $R=(-\infty, \infty)$ is given by

$$
\mu_{\left[a_{\alpha}, b_{\alpha}\right]}(x)= \begin{cases}\alpha, & a \leq x \leq b, \\ 0, & \text { otherwise, }\end{cases}
$$

then $\left[a_{\alpha}, b_{\alpha}\right]$, is called an $\alpha$-level fuzzy interval.

Definition 4 (Zimmermann, 2011). Let $\tilde{B}$ be a FN on $R=(-\infty, \infty)$, and $0 \leq \alpha \leq 1$, then $\alpha$-cut $B(\alpha)$ of $\tilde{B}$ consists of the point $x$ such that $\mu_{\tilde{B}}(x) \geq \alpha$, that is,

$$
B(\alpha)=\left\{x \mid \mu_{\tilde{B}}(x) \geq \alpha\right\} .
$$

Extension Principle (Zimmermann, 2011). Let $\tilde{B}$ be a FN on $R=(-\infty, \infty)$ and $0 \leq \alpha \leq 1$. Suppose that the $\alpha$-cut of $\tilde{B}$ is a closed interval $B(\alpha)=\left[B_{L}(\alpha), B_{U}(\alpha)\right]$, then, we have

or

$$
\tilde{B}=\bigcup_{0 \leq \alpha \leq 1} \alpha B(\alpha)
$$

$$
\mu_{\tilde{B}}(x)=\underset{0 \leq \alpha \leq 1}{\vee} \alpha C_{B(\alpha)}(x)
$$

where

(I) $\alpha B(\alpha)$ is a FN with the MF:

$$
\mu_{\alpha B(\alpha)}(x)= \begin{cases}\alpha, & x \in B(\alpha), \\ 0, & \text { otherwise. }\end{cases}
$$

(II) $C_{B(\alpha)}(x)$ is a CF of $B(\alpha)$, that is,

$$
C_{B(\alpha)}(x)= \begin{cases}1, & x \in B(\alpha), \\ 0, & x \notin B(\alpha) .\end{cases}
$$

From the principle of extension, we obtain

$$
\tilde{B}=\bigcup_{0 \leq \alpha \leq 1} \alpha B(\alpha)=\bigcup_{0 \leq \alpha \leq 1}\left[B_{L}(\alpha)_{\alpha}, B_{U}(\alpha)_{\alpha}\right] .
$$

From Yao and $\mathrm{Wu}$ (2000), we introduce the concept of signed distance of FNs:

Definition 5 (Yao \& Wu, 2000). For any $a$ and $0 \in R, \vartheta(a, 0)=a$ is defined as the signed distance from $a$ to 0 . If $a>0$, then the distance from a to 0 is $a=\vartheta(a, 0)$; If $a<0$, then the distance from $a$ to 0 is $-a=-\vartheta(a, 0)$. Hence, $\vartheta(a, 0)=a$ is called the signed distance from $a$ to 0 . 
From Definition 5, the signed distance of the interval $\left[B_{L}(\alpha), B_{U}(\alpha)\right]$ to 0 is defined as:

$$
\vartheta\left(\left[B_{L}(\alpha), B_{U}(\alpha)\right], 0\right)=\frac{1}{2}\left[\vartheta\left(B_{L}(\alpha), 0\right)+\vartheta\left(B_{U}(\alpha), 0\right)\right]=\frac{1}{2}\left(B_{L}(\alpha)+B_{U}(\alpha)\right) .
$$

For every $\alpha \in[0,1]$, there is a one-to-one mapping between the $\alpha$-fuzzy interval $\left[B_{L}(\alpha)_{\alpha}, B_{U}(\alpha)_{\alpha}\right]$ and the real interval $\left[B_{L}(\alpha), B_{U}(\alpha)\right]$. Also, the $1-$ level fuzzy point $\tilde{0}_{1}$ is a mapping to the real number 0 , and hence, the signed distance of $\left[B_{L}(\alpha)_{\alpha}, B_{U}(\alpha)_{\alpha}\right]$ to $\tilde{0}_{1}$ can be defined as:

$$
\vartheta\left(\left[B_{L}(\alpha)_{\alpha}, B_{U}(\alpha)_{\alpha}\right], \tilde{0}_{1}\right)=\frac{1}{2}\left[\vartheta\left(B_{L}(\alpha), 0\right)+\vartheta\left(B_{U}(\alpha), 0\right)\right]=\frac{1}{2}\left(B_{L}(\alpha)+B_{U}(\alpha)\right) .
$$

Since the above function is continuous on $0 \leq \alpha \leq 1$, then, we have the following definition:

Definition 6 (Yao and $\mathrm{Wu}, 2000$ ). For $\tilde{B} \in \Omega$, the signed distance of $\tilde{B}$ to $\tilde{0}_{1}$ is defined as:

$$
\vartheta\left(\tilde{B}, \tilde{0}_{1}\right)=\int_{0}^{1} \vartheta\left(\left[B_{L}(\alpha)_{\alpha}, B_{U}(\alpha) \alpha\right], \tilde{0}_{1}\right) d \alpha=\frac{1}{2} \int_{0}^{1}\left(B_{L}(\alpha)+B_{U}(\alpha)\right) d \alpha
$$

\section{Notations}

We first introduce some basic notations which are useful in the remainder of the paper: $p$ : Unit retail price;

$c$ : Manufacturer's unit production cost;

$\tilde{D}$ : Uncertain demand;

$\theta$ : Defective rate of products;

$W_{d}$ : Wholesale price per unit with the decentralized channel, $W_{d}>c /(1-\theta)$;

$h$ : Retailer's unit holding cost;

$s$ : Retailer's unit shortage cost;

$R$ : Compensation payment per unit by the manufacturer for the returned good items;

$\lambda$ : Discount rate for per unit under quantity discount with return policy;

$q$ : Retailer's order quantity;

$\varepsilon$ : Increase rate of retailer's profit.

If the demand $x$ is smaller than $q(1-\theta)$, then the inventory is sufficient, an amount of $q(1-\theta)-x$ will be left, and the total profit of the retailer will be $p x-h(q(1-\theta)-x)-W_{d} q(1-\theta)$. Otherwise, if the demand $x$ is greater than $q(1-\theta)$, then the inventory is insufficient by an amount of $x-q(1-\theta)$, the total profit of the retailer will be $p x-s(x-q(1-\theta))-W_{d} q(1-\theta)$ for a given $q$ with $\theta$ defective rate, and the total profit of the retailer will depend on the demand $x$ :

$$
\tilde{\pi}_{r}(x \mid q)= \begin{cases}p x-h(q(1-\theta)-x)-W_{d} q(1-\theta), & x \leq q(1-\theta), \\ p x-s(x-q(1-\theta))-W_{d} q(1-\theta), & x>q(1-\theta) .\end{cases}
$$

The MF of the fuzzy profit $\tilde{\pi}_{r}(x \mid q)$ is the same with that of the demand $x$ (Kao \& Hsu, 2002; Chen \& Ho, 2011). Here we assume that the uncertain demand $\tilde{D}$ is expressed as a TFN (Dubois \& Prade, 1987) (Figure 1). Due to the lack of historical information, the demand for products is usually inaccurate, and the retailer can expect: "the demand for this season will is likely to be $d$ units, not less than $d-\sigma_{1}$ and no more than $d+\sigma_{2}$ ". Especially, if $\sigma_{1}=\sigma_{2}=0$, then $\tilde{D}$ is reduced to the real number $d$. 


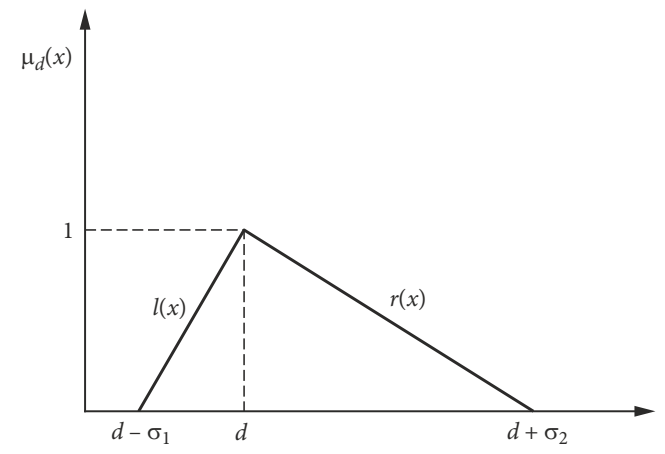

Figure 1. The MF of the demand $\tilde{D}$

The uncertain demand can be described as the following MF:

$$
\mu_{\tilde{D}}(x)= \begin{cases}l(x)=\left(x-d+\sigma_{1}\right) / \sigma_{1}, & d-\sigma_{1} \leq x \leq d, \\ r(x)=\left(d+\sigma_{2}-x\right) / \sigma_{2}, & d<x \leq d+\sigma_{2}, \\ 0, & \text { otherwise. }\end{cases}
$$

\section{Models}

We take into account a return policy for quantity discount between a manufacturer and a retailer in a two-echelon SC with imperfect quality. The manufacturer forecasts the uncertain demand $\tilde{D}$ in a TFN and designs a return policy with quantity discount contract to the retailer. Then the manufacturer supplies the products with imperfect quality to the retailer and the retailer determines $q$ and the products are not examined with $\theta$ defective rate to the customer. The imperfect products are returned to the retailer, and then to the manufacturer, and the manufacturer designs a return price $p$ with the quantity discount $\lambda$ to give an incentive to the retailer (Figure 2).

We first model the decentralized SC channel to solve the optimal $q^{*}$. Then we establish the integrated SC channel to calculate the optimal $q^{* *}$, and demonstrate that the retailer's profit decreases with the integrated channel. In this case, the manufacturer supplies the return policy with quantity discount to give an incentive to the retailer.

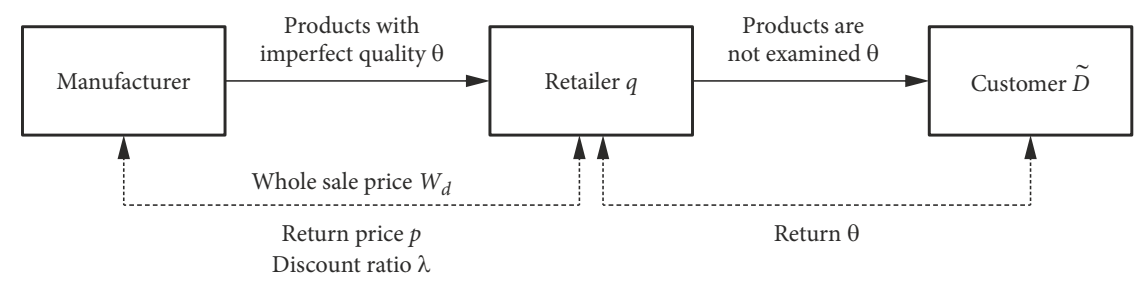

Figure 2. Coordination relationships of SC members 


\subsection{The decentralized channel}

Here we give several basic expressions, where the retailer orders products and the manufacturer provides products in a decentralized channel:

1) The retailer's profit is the differences of the sales volume, the shortage cost, the holding cost, and the purchasing cost: the demand is subjectively believed to be a normal FN $\tilde{D}$.

$\tilde{\pi}_{r}=p \min (q(1-\theta), \tilde{D})-h \max ((q(1-\theta)-\tilde{D}), 0)-s \max (\tilde{D}-q(1-\theta), 0)-W_{d}(1-\theta) q, D \in \tilde{D}_{\alpha}$.

2) The manufacturer's profit is the difference of the purchasing volume and the manufacturing cost:

$$
\pi_{M}=W_{d}(1-\theta) q-c q .
$$

To obtain the retailer's optimal OQ, we use Eq. (10) to express the retailer's fuzzy profit $\vartheta\left(\tilde{\pi}_{r}\right)$. By analyzing the characteristics of the function, the optimal OQ is given. Due to that the MF of the demand $\tilde{D}$ is expressed with $l(x)$ and $r(x)$, in what follows, we discuss the MF of $\tilde{\pi}_{r}(q)$ from two cases $d-\sigma_{1}<q(1-\theta)<d$ and $d<q(1-\theta)<d+\sigma_{2}$ :

Case 1. If $d-\sigma_{1}<q(1-\theta)<d$, then using then the extension principle, the demand $\tilde{D}$ 's MF is $l(x)$, the retailer's fuzzy profit also has the same MF with the demand. The $\alpha$-level sets of fuzzy sales volume, the fuzzy holding quantity and the fuzzy shortage quantity under the decentralized channel can be derived as follows (Zheng et al., 2010):

$$
\begin{aligned}
\{\min (q(1-\theta), \tilde{D})\}(\alpha) & = \begin{cases}{\left[d+(\alpha-1) \sigma_{1}, q(1-\theta)\right],} & \alpha \in\left(0, \alpha_{0}\right], \\
{[q(1-\theta), q(1-\theta)],} & \alpha \in\left(\alpha_{0}, 1\right] .\end{cases} \\
\{\max (q(1-\theta)-\tilde{D}, 0)\}(\alpha) & = \begin{cases}{\left[0, q(1-\theta)-d-(\alpha-1) \sigma_{1}\right],} & \alpha \in\left(0, \alpha_{0}\right], \\
{[0,0],} & \alpha \in\left(\alpha_{0}, 1\right] .\end{cases} \\
\{\max (\tilde{D}-q(1-\theta), 0)\}(\alpha) & = \begin{cases}{\left[0, d-q(1-\theta)-(\alpha-1) \sigma_{2}\right],} & \alpha \in\left(0, \alpha_{0}\right], \\
{\left[d-q(1-\theta)+(\alpha-1) \sigma_{1}, d-q(1-\theta)-(\alpha-1) \sigma_{2}\right],} & \alpha \in\left(\alpha_{0}, 1\right] .\end{cases}
\end{aligned}
$$

where $\alpha_{0}=1+\frac{q(1-\theta)-d}{\sigma_{1}}$. Using the defuzzied value $\vartheta(\tilde{A})=\int_{0}^{1} \frac{1}{2}\left(A_{\alpha}^{L}+A_{\alpha}^{U}\right) d \alpha$, and according to Eqs. (10), (12) and (14)-(16), we get the retailer's expected profit (EP):

$$
\begin{aligned}
& \vartheta\left(\tilde{\pi}_{r}(q)\right)(\alpha)=\int_{0}^{\alpha_{0}} 0.5 p\left(d+(\alpha-1) \sigma_{1}+q(1-\theta)\right) d \alpha+\int_{\alpha_{0}}^{1} 0.5 p(2 q(1-\theta)) d \alpha- \\
& 0.5 h \int_{0}^{\alpha_{0}}\left(q(1-\theta)-d-(\alpha-1) \sigma_{1}\right) d \alpha-0.5 s \int_{0}^{1}\left(d-q(1-\theta)-(\alpha-1) \sigma_{2}\right) d \alpha- \\
& 0.5 s \int_{\alpha_{0}}^{1}\left(\left(d-q(1-\theta)+(\alpha-1) \sigma_{1}\right) d \alpha-W_{d} q(1-\theta) .\right.
\end{aligned}
$$

Simplifying Eq. (17) with the integral method, we obtain the retailer's profit:

$$
\vartheta\left(\tilde{\pi}_{r}(q)\right)=-\frac{(p+h+s)}{4 \sigma_{1}}\left(q(1-\theta)-d+\sigma_{1}\right)^{2}+\left(p+s-W_{d}\right) q(1-\theta)+\frac{s}{4}\left(\sigma_{1}-\sigma_{2}\right)-s d .
$$


In order to get the retailer's optimal OQ, we can calculate the first-order partial derivative and the second order partial derivative $\vartheta\left(\tilde{\pi}_{r}(q)\right)$ with respect to $q$ :

$$
\begin{aligned}
& \frac{d\left(\vartheta\left(\tilde{\pi}_{r}(q)\right)\right.}{d q}=-\frac{(p+h+s)(1-\theta)}{2 \sigma_{1}}\left(q(1-\theta)-d+\sigma_{1}\right)+\left(p+s-W_{d}\right)(1-\theta), \\
& \frac{d^{2}\left(\vartheta\left(\tilde{\pi}_{r}(q)\right)\right.}{d q^{2}}=-\frac{p+h+s}{2 \sigma_{1}}(1-\theta)^{2}<0 .
\end{aligned}
$$

Since the second-order partial derivative $\vartheta\left(\tilde{\pi}_{r}(q)\right)$ with respect to $q$ is smaller than 0 , then the retailer's profit reaches maximum when the first-order partial derivative equals zero, and the optimal OQ is:

$$
q_{l}^{*}=\frac{d-\sigma_{1}}{1-\theta}+\frac{2 \sigma_{1}\left(p+s-W_{d}\right)}{(p+h+s)(1-\theta)} .
$$

Case 2. If $d<q(1-\theta)<d+\sigma_{2}$, then similar to Case 1 , and using the extension principle, the demand $\tilde{D}$ 's MF is $r(x)$, so texhe retailer's fuzzy profit also has the same MF with the demand $\tilde{D}$. Then we have:

$$
\begin{aligned}
\{\min (q(1-\theta), \tilde{D})\}(\alpha) & = \begin{cases}{\left[d+(\alpha-1) \sigma_{1}, q(1-\theta)\right],} & \alpha \in\left(0, \alpha_{0}\right], \\
{\left[d+(\alpha-1) \sigma_{1}, d-(\alpha-1) \sigma_{2}\right],} & \alpha \in\left(\alpha_{0}, 1\right] .\end{cases} \\
\{\max (q(1-\theta)-\tilde{D}, 0)\}(\alpha) & = \begin{cases}{\left[0, q(1-\theta)-d-(\alpha-1) \sigma_{1}\right],} \\
{\left[q(1-\theta)-d+(\alpha-1) \sigma_{2}, q(1-\theta)-d-(\alpha-1) \sigma_{1}\right],} & \alpha \in\left(\alpha_{0}, 1\right] .\end{cases} \\
\{\max (\tilde{D}-q(1-\theta), 0)\}(\alpha) & = \begin{cases}{\left[0, d-q(1-\theta)-(\alpha-1) \sigma_{2}\right],} & \alpha \in\left(0, \alpha_{0}\right], \\
{[0,0],} & \alpha \in\left(\alpha_{0}, 1\right],\end{cases}
\end{aligned}
$$

where $\alpha_{0}=1-\frac{q(1-\theta)-D}{\sigma_{2}}$. Using the defuzzied value $\vartheta(\tilde{A})=\int_{0}^{1} \frac{1}{2}\left(A_{\alpha}^{L}+A_{\alpha}^{U}\right) d \alpha$, and according to Eqs (10), (12), (22), (23) and (24), we get the retailer's EP:

$$
\begin{aligned}
& \vartheta\left(\tilde{\pi}_{r}(q)\right)(\alpha)=\int_{0}^{1} 0.5 p\left(d+(\alpha-1) \sigma_{1}\right) d \alpha+\int_{0}^{\alpha_{0}} 0.5 p q(1-\theta) d \alpha+\int_{\alpha_{0}}^{1} 0.5 p\left(d-(\alpha-1) \sigma_{2}\right) d \alpha- \\
& 0.5 h \int_{0}^{1}\left(q(1-\theta)-d-(\alpha-1) \sigma_{1}\right) d \alpha-0.5 h \int_{\alpha_{0}}^{1}\left(q(1-\theta)-d+(\alpha-1) \sigma_{2}\right) d \alpha- \\
& 0.5 s \int_{0}^{\alpha_{0}}\left(d-q(1-\theta)-(\alpha-1) \sigma_{2}\right) d \alpha-W_{d} q(1-\theta) .
\end{aligned}
$$

Additionally, simplifying Eq. (25) with the integral method, then we have:

$$
\begin{aligned}
& \vartheta\left(\tilde{\pi}_{r}(q)\right)=-\frac{1}{4 \sigma_{2}}(p+h+s)\left(q(1-\theta)-d-\sigma_{2}\right)^{2}-\left(h+W_{d}\right) q(1-\theta)+ \\
& (p+h) d-\frac{1}{4}(p+h)\left(\sigma_{1}-\sigma_{2}\right) .
\end{aligned}
$$

After that, similar to Case 1, we have:

$$
\begin{aligned}
& \frac{d\left(\vartheta\left(\tilde{\pi}_{r}(q)\right)\right.}{d q}=-\frac{(p+h+s)(1-\theta)}{2 \sigma_{2}}\left(q(1-\theta)-d-\sigma_{2}\right)-\left(h+W_{d}\right)(1-\theta), \\
& \frac{d^{2}\left(\vartheta\left(\tilde{\pi}_{r}(q)\right)\right.}{d q^{2}}=-\frac{p+h+s}{2 \sigma_{2}}(1-\theta)^{2}<0 .
\end{aligned}
$$


The retailer's profit reaches maximum when the first-order partial derivative equals zero, and the optimal OQ is:

$$
q_{u}^{*}=\frac{d+\sigma_{2}}{1-\theta}-\frac{2 \sigma_{2}\left(h+W_{d}\right)}{(p+h+s)(1-\theta)} .
$$

Consequently, by Eqs. (21) and (29), we get the optimal OQ in a decentralized channel:

$$
q^{*}= \begin{cases}\frac{d-\sigma_{1}}{1-\theta}+\frac{2 \sigma_{1}\left(p+s-W_{d}\right)}{(p+s+h)(1-\theta)}, & p+s-h-2 W_{d}<0, \\ \frac{d+\sigma_{2}}{1-\theta}-\frac{2 \sigma_{2}\left(h+W_{d}\right)}{(p+h+s)(1-\theta)}, & p+s-h-2 W_{d} \geq 0 .\end{cases}
$$

\subsection{The integrated channel}

Considering an integrated channel where a manufacturer and a retailer work together as a company. They make decisions of their OQ to maximize the profits of the entire SC. First, we represent the fuzzy profit of the total SC, and then defuzzify the profit and calculate the OQ using the optimization theory. We compare the OQ, the EP of the manufacturer and the retailer with different channels, and demonstrate that the OQ, the total SC's EP and the manufacturer's EP increase with the integrated channel, but the retailer's EP decreases with the integrated channel.

The total profit of SC in an integrated channel is:

$$
\left.\tilde{\pi}_{T}=\tilde{\pi}_{r}+\tilde{\pi}_{M}=p \min (q(1-\theta), \tilde{D})-h \max (q(1-\theta)-\tilde{D}), 0\right)-s \max (\tilde{D}-q(1-\theta), 0)-c q .
$$

Similar to Section 4.1, we use Eq. (31) to express the fuzzy total profit of the SC with the integrated channel, and discuss the following two cases:

Case 1. If $d-\sigma_{1}<q(1-\theta)<d$, then similar to Case 1 within the decentralized channel in Section 4.1, we have:

$$
\begin{aligned}
& \vartheta\left(\tilde{\pi}_{T}(q)\right)(\alpha)=\int_{0}^{\alpha_{0}} 0.5 p\left(d+(\alpha-1) \sigma_{1}+q(1-\theta)\right) d \alpha+\int_{\alpha_{0}}^{1} 0.5 p(2 q(1-\theta)) d \alpha- \\
& 0.5 h \int_{0}^{\alpha_{0}}\left(q(1-\theta)-d-(\alpha-1) \sigma_{1}\right) d \alpha-0.5 s \int_{0}^{1}\left(d-q(1-\theta)-(\alpha-1) \sigma_{2}\right) d \alpha- \\
& 0.5 s \int_{\alpha_{0}}^{1}\left((d-q(1-\theta))+(\alpha-1) \sigma_{1}\right) d \alpha-c q,
\end{aligned}
$$

where $\alpha_{0}=1+\frac{q(1-\theta)-d}{\sigma_{1}}$. Then we get the total profit:

$$
\vartheta\left(\tilde{\pi}_{T}(q)\right)(\alpha)=-\frac{(p+h+s)}{4 \sigma_{1}}\left(q(1-\theta)-d+\sigma_{1}\right)^{2}+(p+s) q(1-\theta)-c q+\frac{s}{4}\left(\sigma_{1}-\sigma_{2}\right)-s d .
$$

Then

$$
\begin{aligned}
& \frac{d\left(\vartheta\left(\tilde{\pi}_{T}(q)\right)\right.}{d q}=-\frac{(p+h+s)(1-\theta)}{2 \sigma_{1}}\left(q(1-\theta)-d+\sigma_{1}\right)+(p+s)(1-\theta)-c, \\
& \frac{d^{2}\left(\vartheta\left(\tilde{\pi}_{T}(q)\right)\right.}{d q^{2}}=-\frac{p+h+s}{2 \sigma_{1}}(1-\theta)^{2}<0 .
\end{aligned}
$$


Since the second-order partial derivative $\vartheta\left(\tilde{\pi}_{r}(q)\right)$ with respect to $q$ is smaller than 0 , then the retailer's profit reaches maximum when the first-order partial derivative equals zero, and the optimal OQ is:

$$
q_{l}^{* *}=\frac{d-\sigma_{1}}{1-\theta}+\frac{2 \sigma_{1}(p+s)}{(p+h+s)(1-\theta)}-\frac{2 \sigma_{1} c}{(p+s+h)(1-\theta)^{2}} .
$$

Case 2. If $d<q(1-\theta)<d+\sigma_{2}$, then similar to Case 2 with the decentralized channel in Section 4.1, we have:

$$
\begin{aligned}
& \vartheta\left(\tilde{\pi}_{T}(q)\right)(\alpha)=\int_{0}^{1} 0.5 p\left(d+(\alpha-1) \sigma_{1}\right) d \alpha+\int_{0}^{\alpha_{0}} 0.5 p q(1-\theta) d \alpha+\int_{\alpha_{0}}^{1} 0.5 p\left(d-(\alpha-1) \sigma_{2}\right) d \alpha- \\
& 0.5 h \int_{0}^{1}\left(q(1-\theta)-d-(\alpha-1) \sigma_{1}\right) d \alpha-0.5 h \int_{\alpha_{0}}^{1}\left(q(1-\theta)-d+(\alpha-1) \sigma_{2}\right) d \alpha- \\
& 0.5 s \int_{0}^{\alpha_{0}}\left(d-q(1-\theta)-(\alpha-1) \sigma_{2} d \alpha-c q .\right.
\end{aligned}
$$

We can further solve the OQ with the integrated channel:

$$
q_{u}^{* *}=\frac{d+\sigma_{2}}{1-\theta}-\frac{2 \sigma_{2} h}{(p+h+s)(1-\theta)}-\frac{2 \sigma_{2} c}{(p+h+s)(1-\theta)^{2}} .
$$

Consequently, by Eqs (36) and (38), the optimal OQ with the integrated channel can be written as:

$$
q_{T}^{* *}= \begin{cases}\frac{d-\sigma_{1}}{1-\theta}+\frac{2 \sigma_{1}(p+s)}{(p+s+h)(1-\theta)}-\frac{2 \sigma_{1} c}{(p+h+s)(1-\theta)^{2}}, & p+s-h-2 c /(1-\theta)<0, \\ \frac{d+\sigma_{2}}{1-\theta}-\frac{2 \sigma_{2} h}{(p+h+s)(1-\theta)}-\frac{2 \sigma_{2} c}{(p+h+s)(1-\theta)^{2}}, & p+s-h-2 c /(1-\theta) \geq 0 .\end{cases}
$$

Comparing two different channels of OQ (i.e., the decentralized channel and the integrated channel), we can get the following propositions:

Proposition 1. The optimal OQ of the integrated channel is higher than the optimal OQ of the decentralized channel.

Proof. Comparing the sufficient conditions of Sections 4.1 and 4.2, there are three cases considering OQ as follows:

Case 1. If $p+s-h-2 c /(1-\theta)<0$ and $p+s-h-2 W_{d}<0$, then the optimal OQ with the decentralized channel and the optimal OQ with the integrated channel are:

$$
\begin{aligned}
& q^{*}=q_{l}^{*}=\frac{d-\sigma_{1}}{1-\theta}+\frac{2 \sigma_{1}\left(p+s-W_{d}\right)}{(p+h+s)(1-\theta)} \\
& q_{T}^{* *}=q_{l}^{* *}=\frac{d-\sigma_{1}}{1-\theta}+\frac{2 \sigma_{1}(p+s)}{(p+h+s)(1-\theta)}-\frac{2 \sigma_{1} c}{(p+s+h)(1-\theta)^{2}} .
\end{aligned}
$$

respectively. Also since $W_{d}>c /(1-\theta)$, then $q_{T}^{* *}>q^{*}$.

Case 2. If $p+s-h-2 c /(1-\theta) \geq 0$ and $p+s-h-2 W_{d} \geq 0$, then he optimal OQ with the decentralized channel is:

$$
q^{*}=q_{u}^{*}=\frac{d+\sigma_{2}}{1-\theta}-\frac{2 \sigma_{2}\left(h+W_{d}\right)}{(p+h+s)(1-\theta)} .
$$


and the optimal OQ with the integrated channel is:

$$
q_{T}^{* *}=q_{u}^{* *}=\frac{d+\sigma_{2}}{1-\theta}-\frac{2 \sigma_{2} h}{(p+h+s)(1-\theta)}-\frac{2 \sigma_{2} c}{(p+h+s)(1-\theta)^{2}} .
$$

Similar to Case 1 , since $W_{d}>c /(1-\theta)$, then $q_{T}^{* *}>q^{*}$.

Case 3. If $p+s-h-2 c /(1-\theta) \geq 0$ and $p+s-h-2 W_{d}<0$, then the optimal OQ with the decentralized channel is:

$$
q^{*}=q_{l}^{*}=\frac{d-\sigma_{1}}{1-\theta}+\frac{2 \sigma_{1}\left(p+s-W_{d}\right)}{(p+h+s)(1-\theta)},
$$

and the optimal OQ with the integrated channel is:

$$
q_{T}^{* *}=q_{u}^{* *}=\frac{d+\sigma_{2}}{1-\theta}-\frac{2 \sigma_{2} h}{(p+h+s)(1-\theta)}-\frac{2 \sigma_{2} c}{(p+h+s)(1-\theta)^{2}} .
$$

Also since $d-\sigma_{1}<q_{l}^{*}<d<q_{u}^{* *}<d+\sigma_{2}$, then $q^{*}<q_{T}^{* *}$.

In summary, $q^{*}<q_{T}^{* *}$, and the optimal OQ with the integrated channel is greater than that with the decentralized channel.

Proposition 2. The EP of the entire SC and the manufacturer are higher in the integrated channel than in the decentralized channel.

Proof. For the EP of entire SC $\vartheta\left(\tilde{\pi}_{T}\right)$, the optimal $q_{T}^{* *}$ can make $\vartheta\left(\tilde{\pi}_{T}\right)$ reach the local maximum points, and then $\vartheta\left(\tilde{\pi}_{T}\left(q_{T}^{* *}\right)\right)>\vartheta\left(\tilde{\pi}_{T}\left(q^{*}\right)\right)$.

Additionally, $q^{*}<q_{T}^{* *}$, and $\pi_{M}=W_{d}(1-\theta) q-c q$ is a linear increasing function of $q$, and thus, $\pi_{M}\left(q_{T}^{* *}\right)>\pi_{M}\left(q^{*}\right)$.

Proposition 3. The retailer's EP is lower than that of the decentralized channel under integrated channel.

Proof. Similar to Proposition 2, for the retailer's EP $\vartheta\left(\tilde{\pi}_{r}\right)$, the optimal $q^{*}$ can make $\vartheta\left(\tilde{\pi}_{r}\right)$ reach the local maximum points, and then $\vartheta\left(\tilde{\pi}_{r}\left(q_{T}^{* *}\right)\right)<\vartheta\left(\tilde{\pi}_{r}\left(q^{*}\right)\right)$.

According to Propositions 1-3, the retailer's profit decreases with the typical integrated channel, so the manufacturer has to supply some mechanisms to give an incentive to the retailer, and thus in the next subsection, we shall propose a return policy with OQ to coordinate the supply relationship.

\subsection{Coordination mechanism with the integrated channel}

In order to give an incentive to the retailer to order the products with the quantity $q_{T}^{* *}$, the manufacturer supplies the return price $R$ and the quantity discount rate $\lambda$ for the retailer. Then we have:

$\tilde{\pi}_{r}^{c}(q)=p \min (q(1-\theta), \tilde{D})+R \max ((q(1-\theta)-\tilde{D}), 0)-s \max (\tilde{D}-q(1-\theta), 0)-W_{d}(1-\theta) q \lambda$

and the manufacturer' profit can be written as:

$$
\pi_{M}{ }^{c}=\lambda W_{d}(1-\theta) q-c q-R \max ((q(1-\theta)-\tilde{D}), 0) .
$$

In our study, we assume that the manufacturer promises the retailer a $\varepsilon$ percent discount on the profit, to guarantee the win-win condition, then $\varepsilon$ satisfies the following condition ( $\tau$ is the maximum increasing rate for the retailer): 


$$
\tau=\frac{\vartheta\left(\tilde{\pi}_{T}^{* *}\right)-\vartheta\left(\tilde{\pi}_{T}^{*}\right)}{\vartheta\left(\tilde{\pi}_{r}^{*}\right)} \geq \varepsilon>0
$$

and the retailer's EP can be expressed as:

$$
\begin{aligned}
& (1+\varepsilon) \tilde{\pi}_{r}\left(q^{*}\right)=p \min \left(q^{* *}(1-\theta), \tilde{D}\right)+R \max \left(\left(q^{* *}(1-\theta)-\tilde{D}\right), 0\right)- \\
& s \max \left(\left(\tilde{D}-q^{* *}(1-\theta), 0\right)-W_{d}(1-\theta) q^{* *} \lambda .\right.
\end{aligned}
$$

So the return price and the discount rate $(\lambda, R)$ should satisfy the following:

$$
\begin{aligned}
& \lambda=\frac{p \min \left(q^{* *}(1-\theta), \tilde{D}\right)}{W_{d}(1-\theta) q^{* *}}+\frac{R \max \left(\left(q^{* *}(1-\theta)-\tilde{D}\right), 0\right)}{W_{d}(1-\theta) q^{* *}}- \\
& \frac{s \max \left(\tilde{D}-q^{* *}(1-\theta), 0\right)}{W_{d}(1-\theta) q^{* *}}-\frac{(1+\varepsilon) \tilde{\pi}_{r}\left(q^{*}\right)}{W_{d}(1-\theta) q^{* *}} .
\end{aligned}
$$

In order to obtain $(\lambda, R)$, we need to consider the decentralized channel and the integrated channel, and then similar to Section 4.2, the following three cases should be discussed:

Case 1. If $p+s-h-2 c /(1-\theta)<0$ and $p+s-h-2 W_{d}<0$, then we synthesize Eqs (18), (40), (41), (49) and (50), and transform the return price and the discount rate $(\lambda, R)$ as:

$$
\lambda=\frac{-\frac{(p-R+s)}{4 \sigma_{1}}\left[\left(q_{l}^{* *}(1-\theta)-d-\sigma_{1}\right)^{2}\right]+(p+s) q_{l}^{* *}(1-\theta)+\frac{s}{4}\left(\sigma_{1}-\sigma_{2}\right)-s d-(1+\varepsilon) \vartheta\left(\tilde{\pi}_{r}\left(q_{l}^{*}\right)\right)}{W_{d} q_{l}^{* *}(1-\theta)} .
$$

Case 2. If $p+s-h-2 c /(1-\theta) \geq 0$ and $p+s-h-2 W_{d} \geq 0$, then we synthesize Eqs (26), (42), (43), (49) and (50), and transform the return price and the discount rate $(\lambda, R)$ as:

$$
\lambda=\frac{-\frac{1}{4 \sigma_{2}}(p-R+s)\left(q_{u}^{* *}(1-\theta)-d-\sigma_{2}\right)^{2}-h q_{u}^{* *}(1-\theta)+(p+s) d-\frac{1}{4}(p+h)\left(\sigma_{1}-\sigma_{2}\right)-(1+\varepsilon) \vartheta\left(\tilde{\pi}_{r}\left(q_{u}^{* *}\right)\right)}{W_{d} q_{u}^{* *}(1-\theta)} .
$$

Case 3. If $p+s-h-2 c /(1-\theta) \geq 0$ and $p+s-h-2 W_{d}<0$, then we synthesize Eqs (26), (44), (45), (49) and (50), and transform the return price and the discount rate $(\lambda, R)$ as:

$$
\lambda=\frac{-\frac{1}{4 \sigma_{2}}(p-R+s)\left(q_{u}^{* *}(1-\theta)-d-\sigma_{2}\right)^{2}-h q_{u}^{* *}(1-\theta)+(p+s) d-\frac{1}{4}(p+h)\left(\sigma_{1}-\sigma_{2}\right)-(1+\varepsilon) \vartheta\left(\tilde{\pi}_{r}\left(q_{l}^{*}\right)\right)}{W_{d} q_{u}^{* *}(1-\theta)} .
$$

The manufacturer and the retailer first judge which case in the above comes up to the actual environment, they negotiate so as to get a rational value of $\varepsilon$, and then determine a satisfied set of $(\lambda, R)$ from Eq. (51), (52) or (53).

\subsection{Steps for SC coordination process}

From the above propositions, we get the following steps for the SC coordination with quantity discount and buyback policy. 
Step 1. With the uncertain demand, defective rate, costs, wholesale price and sale price, we calculate the retailer's OQ with different channels $q^{*}$ and $q_{T}^{* *}$, the retailer's EP with decentralized channel $\vartheta\left(\tilde{\pi}_{r}^{*}\right)$, and the channel's EP with different channels $\vartheta\left(\tilde{\pi}_{T}^{*}\right)$ and $\vartheta\left(\tilde{\pi}_{T}^{* *}\right)$.

Step 2. Calculate the maximum growth rate $\tau$ for the retailer with Eq. (48).

Step 3. The manufacturer and the retailer coordinate an appropriately value of $\varepsilon(0<\varepsilon \leq \tau)$ for the integrated channel.

Step 4. Based on the values of costs, price and the defective rate, judge which case is in three cases, then determine a satisfied set of $(\lambda, R)$ from Eq. (51), (52) or (53).

\section{Numerical examples}

To illustrate the proposed solution procedure, in the following, we investigate three cases with the different values of the parameters. $S$ is a new product from the manufacturer $M$ with imperfect quality, $\mathrm{N}$ is a downstream retailer, owing to the lack of statically data, the demand is uncertain, and the retailer $\mathrm{N}$ forecasts the demand with TFN.

Let's assume the uncertain demand: $\tilde{D}=[800,1000,1150]$, the defective rate: $\theta=0.08$, the retailer N's holding cost: $h=12$, the shortage cost: $s=8$, the manufacturer M's cost: $c=12$, and the wholesale price from $\mathrm{M}$ to $\mathrm{N}: W_{d}=20$. In the case where the sale price is $p \in[20,50]$, the optimal order quantities with the decentralized channel and the integrated channel are shown in Figure 3.

As can be seen from Figure 3, the OQ of integrated channel is always higher than that of decentralized channel. When $p \in[20,30], p+s-h-2 c /(1-\theta)<0$ and $p+s-h-2 W_{d}<0$, the OQ conforms to Case 1 in Proposition 1; when $p \in[30,44], p+s-h-2 c /(1-\theta)>0$ and $p+s-h-2 W_{d}<0$, the OQ conforms to Case 3 in Proposition 1 ; when $p \in[44,50]$, $p+s-h-2 c /(1-\theta)>0$ and $p+s-h-2 W_{d}>0$, the OQ conforms to Case 2 in Proposition 1.

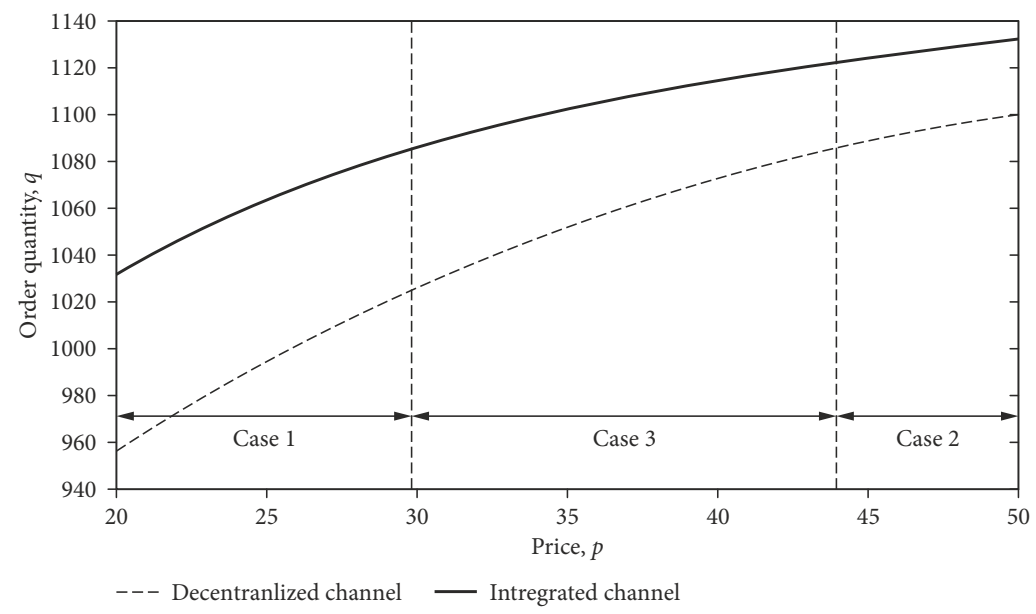

Figure 3. Optimal order quantity 
The EPs of the entire SC and the manufacturer under the integrated channel are greater than that of the decentralized channel, while the EP of retailer has been reduced. We give three examples to discuss the SC coordination problems:

Case 1. When $p=25, p+s-h-2 c /(1-\theta)=-5.08<0$ and $p+s-h-2 W_{d}=-19<0$, the retailer N's OQ increases from 995 to 1062, the total SC's profit increases from 9619 to 9835 , the manufacturer M's profit increases from 6368 to 6797, but the retailer N's EP decreases from 3251 to 3038 . These changes can be seen in Table 1. The typical integrated channel could not stimulate the retailer to increase the OQ.

Table 1. Optimal solutions with the different channels

\begin{tabular}{|l|c|c|}
\hline & Decentralized channel & Integrated channel \\
\hline Retailer's OQ & 995 & 1062 \\
\hline Retailer's EP & 3251 & 3036 \\
\hline Manufacturer's EP & 6368 & 6797 \\
\hline Channel's EP & 9619 & 9833 \\
\hline
\end{tabular}

In order to guarantee the win-win condition, according to Eq. (40), the increase rate $\varepsilon$ of the retailer $\mathrm{N}$ should be smaller than $(9833-9619) / 3251=6.58 \%$, we discuss four different values of $\varepsilon$ : i.e., $0,1 \%, 3.3 \%$ and $6.58 \%$, respectively. The results obtained are shown in Table 2.

Table 2. Quantity discount with return policy in Case 1

\begin{tabular}{|c|c|c|c|c|c|}
\hline$\varepsilon$ & $R$ & $\lambda$ & $\vartheta\left(\tilde{\pi}_{r}\right)$ & $\vartheta\left(\tilde{\pi}_{M}\right)$ & $\vartheta\left(\tilde{\pi}_{T}\right)$ \\
\hline 0 & 0 & 0 & 3251 & 6368 & 9619 \\
\hline 1 & 5 & 0.95 & 3286 & 6549 & 9835 \\
\hline 3.3 & 10 & 0.97 & 3358 & 6477 & 9835 \\
\hline 6.58 & 12 & 0.98 & 3464 & 6369 & 9835 \\
\hline
\end{tabular}

From Table 2, we can see the total SC profit is optimal with the different values of $(\lambda, R)$, and the retailer's EP and the manufacturer's EP also increase.

Case 2. When $p=45, p+s-h-2 c /(1-\theta)=14.92>0$ and $p+s-h-2 W_{d}=1>0$, the retailer N's OQ increases from 1089 to 1124 , the total SC' EP increases from 21740 to 21850 , the manufacturer M's profit increases from 28713 to 29043, but the retailer N's EP decreases from 6973 to 6715 . The typical integrated channel could not stimulate the retailer to increase the OQ. The results obtained are shown in Table 3.

Table 3. Optimal solutions in Case 2

\begin{tabular}{|l|c|c|}
\hline & Decentralized channel & Integrated channel \\
\hline Retailer's OQ & 1089 & 1124 \\
\hline Retailer's EP & 21740 & 21850 \\
\hline Manufacturer's EP & 6973 & 7196 \\
\hline Channel's EP & 28713 & 29043 \\
\hline
\end{tabular}


In order to guarantee the win-win condition, according to Eq. (40), the increase rate $\varepsilon$ of the retailer $\mathrm{N}$ should be smaller than $(29043-28713) / 21740=1.52 \%$, we discuss five different values of $\varepsilon$ : i.e., $0,0.76 \%, 1 \%$, and $1.52 \%$, respectively. The results obtained are shown in Table 4.

From Table 4, we can see the total SC profit is optimal with the different values of $(\lambda, R)$, and the retailer's EP and the manufacturer's EP also increase.

Table 4. Quantity discount with return policy in Case 2

\begin{tabular}{|c|c|c|c|c|c|}
\hline$\varepsilon$ & $R$ & $\lambda$ & $\vartheta\left(\tilde{\pi}_{r}\right)$ & $\vartheta\left(\tilde{\pi}_{M}\right)$ & $\vartheta\left(\tilde{\pi}_{T}\right)$ \\
\hline 0 & 0 & 0 & 21740 & 6973 & 28713 \\
\hline 0.76 & 3 & 0.94 & 21905 & 7138 & 29043 \\
\hline 1 & 5 & 0.95 & 21957 & 7086 & 29043 \\
\hline 1.52 & 8 & 0.97 & 22070 & 6973 & 29043 \\
\hline
\end{tabular}

Case 3. When $p=35, p+s-h-2 c /(1-\theta)=4.92>0$ and $p+s-h-2 W_{d}=-9<0$, the retailer N's OQ increases from 1051 to 1102 , the total SC' profit increases from 19149 to 19320 , the manufacturer M's profit increases from 6729 to 7050, but the retailer N's EP decreases from 12420 to 12270 . The typical integrated channel could not stimulate the retailer to increase the OQ (see Table 5).

Table 5. Optimal solutions in Case 3

\begin{tabular}{|l|c|c|}
\hline & Decentralized channel & Integrated channel \\
\hline Retailer's OQ & 1051 & 1102 \\
\hline Retailer's EP & 12420 & 12270 \\
\hline Manufacturer's EP & 6729 & 7050 \\
\hline Channel's EP & 19149 & 19320 \\
\hline
\end{tabular}

In order to guarantee the win-win condition, according to Eq. (40), the increase rate $\varepsilon$ of the retailer $\mathrm{N}$ should be smaller than $(19320-19149) / 12420=1.38 \%$, we discuss five different values of $\varepsilon$ : i.e., $0,1 \%, 1.38 \%$, respectively. The results obtained are shown in Table 6 .

Table 6. Quantity discount with return policy in Case 3

\begin{tabular}{|c|c|c|c|c|c|}
\hline$\varepsilon$ & $R$ & $\lambda$ & $\vartheta\left(\tilde{\pi}_{r}\right)$ & $\vartheta\left(\tilde{\pi}_{M}\right)$ & $\vartheta\left(\tilde{\pi}_{T}\right)$ \\
\hline 0 & 0 & 0 & 12420 & 6729 & 19149 \\
\hline 1 & 4 & 0.96 & 12544 & 6776 & 19320 \\
\hline 1.38 & 6 & 0.99 & 12591 & 6729 & 19320 \\
\hline
\end{tabular}

From Table 6, we can see the total SC profit is optimal with different values of $(\lambda, R)$, and the EP of the retailer and the manufacturer will also increase. 


\section{Conclusions}

We explored the SC consisting of a producer and a retailer. Consider that the manufacturer's products have a certain degree of quality defects, and the manufacturer provides a quantity discount return police. A fuzzy planning model for decentralized SC and integrated SC was constructed. Using the fuzzy programming theory method based on probability distribution to solve the proposed models, the research shows that:

1) The optimal OQ of the integrated channel is higher than the optimal OQ of the decentralized channel.

2) The EPs of the entire SC and the manufacturer are higher in the integrated channel than those in the decentralized channel.

3) The retailer's EP is lower than that of the decentralized channel under integrated channel.

The main contribution is to develop a SC return policy model with product quality defects and quantity discounts, which is based on the possibility fuzzy optimization algorithm. According to a typical integrated channel, the retailer's EP will decrease compared to the decentralized channel, and both parties can not agree to the contract. To solve this issue, we proposed a return policy with quantity discount to coordinate the relationship between the supplier and retailer. The corresponding coordination steps were also given. Furthermore, we adopted the optimization theory to maximize the participants' profits and provided some numerical examples to illustrate the optimal solutions.

This paper only considered the combination contract of quantity discount and return of the two-echelon SC under the uncertainty of demand. In management practices, more and more companies need to consider the operations of multinational SCs. In contrast to a county's domestic SC, the multinational SC must also consider the impact of international finance, international trade, and other factors on the contractual parameters in the formulation of contracts. In addition, the three-echelon SC is also a common form of SC in reality. Coordinating the three-echelon SC will be more difficult than coordinating the two-echelon SC, and the research will be more complicated. Therefore, in the future, we will study the threeechelon SC and the form of portfolio contract under the multinational corporation's SC.

\section{Acknowledgements}

The work was supported by the National Natural Science Foundation of China (Nos. 71771155, 71571123).

\section{References}

Ameli, M., Mirzazadeh, A., \& Shirazi, M. A. (2011). Economic order quantity model with imperfect items under fuzzy inflationary conditions. Trends in Applied Science Research, 6(3), 294-303. https://doi.org/10.3923/tasr.2011.294.303

Bhaya, S., Pal, M., \& Nayak, P. K. (2014). Intuitionistic fuzzy optimization technique in EOQ model with two types of imperfect quality items. Advanced Modeling and Optimization, 16(1), 33-50. 
Cachon, G. P. (2003). Supply chain coordination with contracts. In S. Graves, \& T. de Kok (Eds.), Handbooks in operations research and management science: Supply chain management (Chapter 11). North Holland, Amsterdam. https://doi.org/10.1016/S0927-0507(03)11006-7

Cachon, G. P., \& Lariviere, M. A. (2000). Supply chain coordination with revenue sharing contracts: Strengths and limitation (Working Paper). The Wharton School of Business, University of Pennsylvania, Philadelphia.

Chang, H. C. (2004). An application of fuzzy sets theory to the EOQ model with imperfect quality items. Computer \& Operations Research, 31(12), 2079-2092. https://doi.org/10.1016/S0305-0548(03)00166-7

Chen, L. H., \& Kang, F. S. (2010). Coordination between vendor and buyer considering trade credit and items of imperfect quality. International Journal Production Economics, 123(1), 52-61. https://doi.org/10.1016/j.ijpe.2009.06.043

Chen, S. H., Wang, C. C., \& Chang, S. M. (2007). Fuzzy economic production quantity model for items with imperfect quality. International Journal of Innovative computing, Information and Control, 3(1), 85-95.

Chen, S. P., \& Ho, Y. H. (2011). Analysis of the newsboy problem with fuzzy demands and incremental discounts. International Journal Production Economics, 129(1), 169-177. https://doi.org/10.1016/j.ijpe.2010.09.014

Chung, K. J., Her, C. C., \& Lin, S. D. (2009). A two-warehouse inventory model with imperfect quality production processes. Computer \& Industrial Engineering, 56(1), 193-197. https://doi.org/10.1016/j.cie.2008.05.005

Das, P., De, S. K., \& Sana, S. S. (2015). An EOQ model for time dependent backlogging over idle time: A step order fuzzy approach. International Journal of Applied and Computational Mathematics, 1(2), 171-185. https://doi.org/10.1007/s40819-014-0001-y

De, S. K., \& Sana, S. S. (2015). An alternative fuzzy EOQ model with backlogging for selling price and promotional effort sensitive demand. International Journal of Applied and Computational Mathematics, 1(1), 69-86.

Dolan, R. J. (1987). Quamtity discounts: Managerial issues and research opportunities. Marketing Science, 6(1), 1-27. https://doi.org/10.1287/mksc.6.1.1

Dubois, D., \& Prade, H. (1987). The mean value of a fuzzy number. Fuzzy Sets and Systems, 24(3), 279300. https://doi.org/10.1016/0165-0114(87)90028-5

Emmons, H., \& Gibert, S. M. (1998). Note. The role of returns policies in pricing and inventory decisions for catalogue goods. Management Science, 44(2), 276-283. https://doi.org/10.1287/mnsc.44.2.276

Hsu, J. T., \& Hsu, L. F. (2013). An EOQ model with imperfect quality items, inspection errors, shortage backordering, and sales returns. International Journal of Production Economics, 143(1), 162-170. https://doi.org/10.1016/j.ijpe.2012.12.025

Hsu, J., \& Hsu, L. (2014). A supplement to an EOQ model with imperfect quality items, inspection errors, shortage backordering, and sales return. International Journal of Industrial Engineering Computations, 5(2), 199-210. https://doi.org/10.5267/j.ijiec.2014.2.001

Hu, J. S., Zheng, H., Guo, C. Y., \& Ji, Y. P. (2010). Optimal production run length with imperfect production processes and backorder in fuzzy random environment. Computer \& Industrial Engineering, 59(1), 9-15. https://doi.org/10.1016/j.cie.2010.01.012

Jaber, M. Y., Goyal, S. K., \& Imran, M. (2008). Economic production quantity model for items with imperfect quality subject to learning effects. International Journal Production Economics, 115(1), 143-150. https://doi.org/10.1016/j.ijpe.2008.05.007

Kao, C., \& Hsu, W. K. (2002). A single period inventory model with fuzzy demand. Computers and Mathematics with Applications, 43(6-7), 841-848. https://doi.org/10.1016/S0898-1221(01)00325-X 
Kazemi, N., Olugu, E. U., Abdul-Rashid, S. H., \& Ghazilla, R. A. B. R. (2015a). Development of a fuzzy economic order quantity model for imperfect quality items using the learning effect on fuzzy parameters. Journal of Intelligent \& Fuzzy Systems, 28(5), 2377-2389. https://doi.org/10.3233/IFS-141519

Kazemi, N., Shekarian, E., Cárdenas-Barrón, L. E., \& Olugu, E. U. (2015b). Incorporating human learning into a fuzzy EOQ inventory model with backorders. Computers \& Industrial Engineering, 87, 540-542. https://doi.org/10.1016/j.cie.2015.05.014

Khan, M., Jaber, M. Y., \& Bonney, M. (2011). An economic order quantity for items with imperfect quality and inspection errors. International Journal Production Economics, 133(1), 113-118. https://doi.org/10.1016/j.ijpe.2010.01.023

Khan, M., Jaber, M. Y., \& Wahab, M. I. M. (2010). Economic order quantity model for items with imperfect quality with learning in inspection. International Journal Production Economics, 124(1), 87-96. https://doi.org/10.1016/j.ijpe.2009.10.011

Kumar, R. S., Tiwari, M. K., \& Goswami, A. (2016). Two-echelon fuzzy stochastic supply chain for the manufacturer-buyer integrated production-inventory system. Journal of Intelligent Manufacturing, 27(4), 875-888. https://doi.org/10.1007/s10845-014-0921-8

Kurdhi, N. A., Lestari, S. M. P., \& Susanti, Y. (2015). A fuzzy collaborative supply chain inventory model with controllable setup cost and service level constraint for imperfect items. International Journal of Applied Management Science, 7(2), 93-122. https://doi.org/10.1504/IJAMS.2015.069265

Lin, T. Y. (2010). An economic order quantity with imperfect quality and quantity discounts. Applied Mathematical Modelling, 34(10), 3158-3165. https://doi.org/10.1016/j.apm.2010.02.004

Lin, T. Y., \& Yeh, D. H. (2010). Optimal coordination policy for supply chain system under imperfect quality consideration. Journal of Marine Science and Technology, 18(3), 449-457.

Maddah, B., \& Jaber, M. Y. (2008). Economic order quantity for items with imperfect quality: Revisited. International Journal Production Economics, 112(2), 808-815. https://doi.org/10.1016/j.ijpe.2007.07.003

Mahata, G. C., \& Goswami, A. (2013). Fuzzy inventory models for items with imperfect quality and shortage backordering under crisp and fuzzy decision variables. Computers \& Industrial Engineering, 64(1), 190-199. https://doi.org/10.1016/j.cie.2012.09.003

Manna, A. K., Dey, J. K., \& Mondal, S. K. (2014). Three-layer supply chain in an imperfect production inventory model with two storage facilities under fuzzy rough environment. Journal of Uncertainty Analysis and Applications, 2(1), 17. https://doi.org/10.1186/s40467-014-0017-1

Pal, S., Mahapatra, G. S., \& Samanta, G. P. (2016). A three-layer supply chain EPQ model for priceand stock-dependent stochastic demand with imperfect item under rework. Journal of Uncertainty Analysis and Applications, 4(1), 10. https://doi.org/10.1186/s40467-016-0050-3

Rad, M. A., Khoshalhan, F., \& Glock, C. H. (2014). Optimizing inventory and sales decisions in a twostage supply chain with imperfect production and backorders. Computers \& Industrial Engineering, 74, 219-227. https://doi.org/10.1016/j.cie.2014.05.004

Roy, A., \& Samanta, G. P. (2009). Fuzzy continuous review inventory model without backorder for deteriorating items. Electronic Journal of Applied Statistical Analysis, 2(1), 58-66.

Roy, A., Maity, K., kar, S., \& Maiti, M. (2009). A production-inventory model with remanufacturing for defective and usable items in fuzzy-environment. Computer \& Industrial Engineering, 56(1), 87-96. https://doi.org/10.1016/j.cie.2008.04.004

Salameh, M. K., \& Jaber, M. Y. (2000). Economic production quantity model for items with imperfect quality. International Journal Production Economics, 64(1-3) 59-64. https://doi.org/10.1016/S0925-5273(99)00044-4

Sana, S. S. (2011). A production-inventory model of imperfect quality products in a three-layer supply chain. Decision Support Systems, 50(2), 539-547. https://doi.org/10.1016/j.dss.2010.11.012 
Taleizadeh, A. A., Noori-daryan, M., \& Tavakkoli-Moghaddam, R. (2015). Pricing and ordering decisions in a supply chain with imperfect quality items and inspection under buyback of defective items. International Journal of Production Research, 53(15), 4553-4582.

https://doi.org/10.1080/00207543.2014.997399

Wang, W. T., Wee, H. M., Cheng, Y. L., Wen, C. L., \& Cárdenas-Barrón, L. E. (2015). EOQ model for imperfect quality items with partial backorders and screening constraint. European Journal of Industrial Engineering, 9(6), 744-773. https://doi.org/10.1504/EJIE.2015.074384

Yao, J. S., \& Wu, K. (2000). Ranking fuzzy numbers based on decomposition principle and signed distance. Fuzzy Sets and Systems, 116(2), 275-288. https://doi.org/10.1016/S0165-0114(98)00122-5

Yu, Y., \& Jin, T. D. (2011). The return policy model with fuzzy demands and asymmetric information. Applied Soft Computing, 11(2), 1669-1678. https://doi.org/10.1016/j.asoc.2010.05.004

Zadeh, L. A. (1965). Fuzzy sets. Information and Control, 8(3), 338-353. https://doi.org/10.1016/S0019-9958(65)90241-X

Zheng, H., Liu, J. C., \& Wang, W. (2010). Supply chain coordination for fuzzy newsboy problem with imperfect quality. International Journal of Information and Management Science, 21(2), 157-175.

Zimmermann, H. J. (2011). Fuzzy set theory and its applications ( $4^{\text {th }}$ ed.). Boston: Kluwer Nijhoff. 